\title{
V.
}

\section{Ueber die pathologisch-anatomischen Verände- rungen in den Knochen wachsender Thiere unter dem Einfluss minimaler Phosphordosen.}

(Aus dem Pathologisch-anatomischen Institut in St. Petersburg.)

\author{
Experimentelle Arbeit \\ von Dr. A. Kissel, \\ Privatdocenten der Kinderheilkunde an der Universität zu Moskal, \\ älterem Ordinator am $\mathbf{S t}$. Olga Kinderspitale.
}

Die ersten mehr oder weniger genauen Angaben über die Veränderungen, welche Phosphor im thierischen Organismus hervorbringt, finden wir erst im zweiten Viertel unseres Jahrhunderts, wo in der Literatur einige Fälle einer acuten Vergiftung anzutreffen sind, was augenscheinlich durchaus nicht zufällig mit der Einführung des Phosphors in grossen Massen in die Technik zusammenfällt, - gerade damals beginnt die Gründung einiger Phosphorzündholzfabriken. Zum ersten Male wurde die acute Phosphorvergiftung von Schneider in Deutschland beschrieben $^{1}$ ), wo bis zum Jahre 1843 nur 4 ähnliche Fälle bekannt wurden; seitdem kommen dieselben immer häufiger vor, so dass in der ausführlichen Arbeit Levin's schon 44 Fälle angeführt werden. Das sind die am meisten wahrscheinlichen aus den zu jener Zeit in der Literatur etwa über 100 berichteten ${ }^{2}$ ).

In der dieser Arbeit beigegebenen Versuchstabelle fanden wir keine Notiz über Veränderungen im Knochensystem. Wie es auch zu erwarten war, wurde die ganze Aufmerksamkeit bei den Sectionen auf die Veränderungen der lebenswichtigen Organe gerichtet, und es war hauptsächlich die fettige Degeneration der Leber, welche die allgemeine Aufmerksamkeit der Gelehrten auf sich lenkte, um so mehr als diese Degeneration

1) Munk und Leiden, Die acute Phosphorvergiftung. Berlin 1865.

2) Studien über Phosphorvergiftung. Dieses Archiv. Bd.21. 1861. 
bei jeder Section sofort in die Augen sprang. So bemerkte schon Hauff, dass er diese Degeneration 11 mal unter 12 Vergiftungsfällen beobachtet habe. Veränderungen in den Knochen erwähnte einstweilen niemand, obwohl schon lange vorher (im Jahre 1845) die erste Beobachtung über die Erkrankung des Unterkiefers bei Arbeitern der Zündholzfabriken gemacht wurde. Die Sache ist aber die, dass zur Section nur acute Vergiftungsfälle kamen, wo also eine Veränderung im Knochensystem schwer zu erwarten war. Was aber die Veränderungen, die durch acute Phosphorvergiftung hervorgerufen werden, betrifft, so wurden sie schon in früherer Zeit gut beschrieben: fast alle Forscher nebmlich stimmen darin überein, dass der Phosphor in mehr oder weniger kurzer Zeit eine fettige Degeneration der Leber, der Nieren, des Magens, des Herzens, der willkürlichen und glatten Muscalatur und sogar des Lungenparenchyms hervorruft; zahlreiche Hämorrhagien wurden im ganzen Körper gefunden. Aeusserst interessant ist die schon von Diez ${ }^{1}$ im Jahre 1845 gemachte Beobachtung, dass der specifischen Erkrankung des Unterkiefers nur Leute mit cariösen Zähnen unterworfen sind, - eine Thatsache, mit der auch andere Autoren übereinstimmen, die jedoch, ich weiss nicht weshalb, die Aufmerksamkeit der späteren Forscher weniger auf sich zog.

Bibra, der die in der Literatur bekannten Angaben über die Wirkung des Phosphors auf den thierischen Organismus sehr ungenügend fand, stellte (1847) eine ganze Reihe von Experimenten an. Bei 5 Kaninchen, bei Katzen und einigen Fröschen rief er eine acute Phosphorvergiftung hervor, indem er grosse Stücke des Giftes in den Magen einführte, oder dasselbe in das Wasser warf, in welchem Fische sich befanden. Dabei that er der Wirkungen auf das Knochensystem mit keinem Worte Erwähnung. Als er später künstlich eine specifische Erkrankung der Knochen hervorrufen wollte, unterwarf er Kaninchen in besonders dazu eingerichteten Zellen der Wirkung des Phosphordampfes, wobei in einer Reihe der Versuche eine acute Phosphorvergiftung entstand, in der anderen eine chronische. Die Zahl der diesen Experimenten unterworfenen Thiere betrug mehr als 30. Der Autor

1) Bibra und Geist, S. 59 . 
protocollirt nur einige Versuche. Bei Anführung der Resultate, welche er bei der acuten Vergiftung gewann, lässt er sich betreffs des Knochensystems folgendermaassen aus: Bei allen Thieren, die zur Untersuchung genommen wurden, begegnete man nicht den kleinsten Veränderungen im Knochensystem. Die untersuchten Röhrenknochen, sowie die Knochen des Schädels erwiesen sich vollkommen gesund und an dem Periost war nicht einmal eine Spur von Entzündung zu entdecken. Was die Fälle von chronischer Vergiftung betrifft, so wurden auch hier alle Knochen in normalem Zustande gefunden. Bibra fand in den inneren Organen alle diejenigen Veränderungen, welche auch bei Menschen beschrieben wurden, nur im Kieferknochen gelang es ihm niemals, auch nur Spuren eines specifischen Prozesses zu bemerken. In Anbetracht der oben erwähnten Meinung von Diez, dass die Erkrankung des Unterkiefers in einiger Beqiehung zu Erkrankung der Zähne (Freiliegen des Periosts durch Zahnlücken oder wenigstens stark cariöse Zähne selbst) stehe, fing Bibra an, bei gesunden Kaninchen die Zähne zu extrahiren, was ihm indessen misslang: entweder wurden die Zähne gebrochen oder es kam zur Fractur des Unterkiefers selbst; wenn es gelang einen Zahn unverletzt zu extrahiren, so kam dabei doch eine Fractur des Unterkiefers vor. Zwei Kaninchen mit extrahirten Zähnen (und gebrochenem Unterkiefer) wurden einer chronischen Vergiftung mit Phosphordämpfen unterworfen. An der Bruchstelle wurde zwar eine Ablagerung ziemlich lockerer Osteophyten rings um den Callus erreicht, welche nach der Meinung des Forschers bis zu einem gewissen Grade an die Erkrankung des Unterkiefers bei den Arbeitern der Zündholzfabriken erinnerte, aber die Osteophyten hatten nicht jenen engen Zusammenhang mit dem Knochen, welchen er bei Menschen beobachtete.

In der Abhandlung von Senftleben ${ }^{1}$ ), der sich mehr für das Vorhandensein von freiem Phosphor im Blute acut vergifteter Thiere interessirte, finden wir nur im Protocoll eines einzigen Versuchs (No. 10) kurze Bemerkungen über Veränderungen im Knochensystem: eine unbedeutende Trübung im Knorpel des

1) Ueber die Erscheinungen und den anatomiscben Befund bei der Pbosphorvergiftung. Dieses Archiv. Bd. 36, 1866. 
Schultergelenks, wobei in den näher zur Oberfläche liegenden Knorpelzellen Fettkügelchen gefunden wurden; die Markräume der Epiphysen waren mit Blut überfüllt, an einigen Stellen sah man Theilung der Kerne der Knochenmarkzellen.

Wegner ${ }^{1}$ ), welcher findet, dass die Nachrichten über Veränderungen, die im Organismus durch acute Phosphorvergiftung hervorgerufen werden, genügen, macht darauf aufmerksam, dass wir verhältnissmässig wenig über die Wirkungen kleiner Phosphordosen wissen, die in den Organismus im Verlaufe von Wochen oder gar von Monaten eingeführt werden. Indem er die Arbeit von Bibra erwähnt, findet er die Resultate derselben wenig befriedigend, indem er hauptsächlich die merkwürdige Erkrankung des Unterkiefers dabei im Auge hat, deren Ursache nach wie vor völlig unaufgeklärt blieb. Als nächster Ausgangspunkt für die Ergründung dieser Frage diente ihm der ungewöhnliche Krankheitsverlauf bei einem 18jährigen Gesellen, der einen starken Hieb auf die Wade bekam. Die $W$ unde nahm bald einen gangränösen Charakter an, welcher auf das Periost überging, das sich auf einer grossen Strecke abhob; die vorgenommene Amputation verhinderte nicht den lethalen Ausgang. Als er erfuhr, dass dieser Arbeiter seit seinem 4. Lebensjahre in einer Zündholzfabrik gearbeitet und dennoch niemals davon gelitten hatte, sondern als ein vollkommen gesunder Mensch erschien, und als auch die Untersuchung des Unterkiefers und der inneren Organe vollständig negative Resultate gab (auf dieses Alles achtete Wegner unbegreiflicher Weise sehr wenig), er andererseits nicht im Stande war, sich den acuten bösartigen Verlauf dieses Falles zu erklären, stellte er die eben erwähnten Thatsachen in ursächliche Beziehung. Und doch war bisher in keinem der unzähligen beschriebenen acuten und chronischen Vergiftungsfälle ein Knochenleiden ausser dem des Unterkiefers erwähnt worden; ja auch in seinen eigenen Versuchen mit acuter Vergiftung gelang es ihm nicht, irgend welche Veränderungen in den Knorpeln und Knochen zu finden. Um sich noch mehr von der Richtigkeit seiner Voraussetzung zu überzeugen, unternahm er eine Reihe von Versuchen in dieser Richtung an Kaninchen, indem er sie im Laufe

1) Der Einfluss des Phosphors auf den Organismus. Dieses Archiv. Bd. 35. 1872.

Archiv f. pathol. Anat. Bd.144. Hft.1. 
von Wochen und theilweise auch von Monaten mit Phosphor fütterte (die Zahl der Versuche ist nicht angegeben), wobei er sich überzeugen musste, dass seine ursprüngliche Vermuthung sich nicht bestätigte. Kein einziges Mal kam eine Erkrankung des Periosts vor. Nichtsdestoweniger setzte er die Versuche fort und constatirte dabei eine Reihe von interessanten Thatsachen. Wir wollen einen Theil dieser, soweit sie das Knochensystem betreffen, näher darlegen.

In einer Versuchsreihe untersuchte er den örtlichen Einfluss der Phosphordämpfe direct auf das Periost verschiedener Knochen, wobei (die Zahl der Experimente ist auch hier nicht angegeben) augenscheinlich das wirklich stark gereizte Periost eine bedeutend grössere und dichtere Anschwellung hervorbringt, als wenn man sich der Phosphordämpfe nicht bedienen würde. Wegner aber fügte hiebei hinzu, dass beim inneren Gebrauch von Phosphor die Wunden (das Entblössen des Periosts) ebenso schnell heilen, wie unter gewöhnlichen Umständen, so dass der Phosphor offenbar ohne Einfluss auf das Periost bleibt. Zum Zwecke der Ermittelung der Wirkung von kleinen Dosen von Phosphor auf das Knochensystem im Allgemeinen stellte $W$ egner an verschiedenen Thieren eine sehr grosse Zahl von Versuchen (wie viele, ist nicht angegeben) an. $\mathrm{Zu}$ den Versuchen wurden wachsende und schon erwachsene Thiere genommen, wobei die bei beiden gefundenen Erscheinungen etwas variirten. An den Knochen der jungen Thiere zeigten sich die bedeutendsten Veränderungen in der Zone, wo sich der neue Knochen aus dem Knorpel bildet; überall hier wurde das gewöhnliche spongiöse, breitmaschige, viel Knochenmark enthaltende Gewebe durch ein Gewebe ersetat, das durch Gleichmässigkeit, Compactheit und Dichtigkeit ganz an die corticale Lage des Knochens erinnert; schon das unbewaffnete Auge sieht es in Gestalt eines deutlich bemerkbaren Streifens, der der Kürze wegen von dem Autor Phosphorschicht genannt wurde. Bei der mikroskopischen Untersuchung erweist sich diese Lage als aus gut entwickelter Knochensubstanz bestehend. Hier giebt es keine grossen Markräume mehr, da diese sich bis zu der gewöhnlichen Grösse Haversischer Kanäle der compacten Knochenmasse verkleinert haben, um welche herum Spuren eines lamellösen Baues zu sehen sind. Bei fortdauernder Fütterung geht es endlich 
so weit, dass z. B. in den Diaphysenenden alles normale spongiöse Gewebe durch compactes Knochengewebe ersetzt wird, aber bis zur völligen Compactheit der Röhrenknochen (d. h. bis zur Erfüllung des Markkanals durch compactes Gewebe) kommt es, wenigstens bei Kaninchen und Hunden, nicht. Auch das Knochengewebe, das sich zur Zeit der Fütterung mit Phosphor aus Periost entwickelt, bleibt nicht ohne Veränderung. Es erweist sich bei der Untersuchung unter dem Mikroskop gleichfalls viel dichter. Was das corticale Gewebe, das sich vor dem Anfange der Versuche bildete, betrifft, so sklerosirt es bei fortdauernder Fütterung mit Phosphor, namentlich bei relativ starken Dosen, zum zweiten Mal, wobei eine ziemlich grosse Einengung der Haversischen Kanäle entsteht; bis zu ihrer vollen Ausfüllung kommt es aber gewöhnlich nicht.

Da Wegner sich zum Ziele gesetzt hatte, diejenigen Veränderungen $z \mathfrak{u}$ erforschen, welche entstehen würden, wenn das Thier sehr lange mit Phosphor gefüttert würde, z. B. von der Geburt an bis zur Vollendung des Wachsthums, so nahm er die grössten Würfe von Hunden und Kaninchen (hier kommt wieder die gewöhnliche Ungenauigkeit der Angaben, so dass es unbekannt bleibt, wie viel Thiere zur Untersuchung genommen worden sind) und fütterte die eine Hälfte mit kleinen Phosphordosen, während die zweite unter den üblichen Verhältnissen verblieb. Wie er selbst gesteht, gelang ihm aber diese Reihe von Versuchen gar nicht, da gerade alle diejenigen Thiere, die keinen Phosphor erhalten hatten, umkamen. Bezüglich der am Leben gebliebenen drückt sich der Autor sehr unbestimmt aus; es schien ihm, als ob bei ihnen das Knochensystem und die Musculatur ein stärkeres Wachsthum zeigten; er zweifelt aber, ob schliesslich eine grössere Gewebsmasse dadurch entstand. Was dann die Knochen älterer Thiere betrifft, so wurden bei längerem Füttern mit Phosphor folgende Veränderungen gefunden: das spongiöse Gewobe wird compacter durch Verdickung der Knochenbalken, aber bis zur vollen Sklerose des Gewebes kommt es nicht. Auch das compacte Gewebe wird dichter in Folge einer Verengung der Gefässkanäle; endlich verengt sich die Markhöhle merklich in Folge von Bildung neuer Knochenschichten aus den peripherischen Theilen des Knochenmarks, so dass bei unverändertem 
Volumen des Knochens die Wände des Markkanals bedeutend dicker werden. Besonders grosse Dimensionen nimmt dieser Prozess bei Hühnern an, bei welchen es bis zur wirklichen Ausfüllung der Markhöhle durch ächte Knochenmasse kam.

Endlich in einer dritten Reihe von Experimenten, in welchen er die Veränderung in der Neubildung der Knochenmassen bei Brüchen, intraperiostealen Resectionen und periostealen Transplantationen untersuchte, fand er, dass der Gebrauch von kleinen Phosphordosen diese Prozesse bedeutend veränderte. Durch zahlreiche Versuche kam er zu der Ueberzeugung, dass das traumatisch verletzte Periost dabei eine umfangreiche dichtere und dickere Knochenmasse producirt und dass bei Fracturen der Callus die Consistenz des Elfenbeins erreicht, ganz abgesehen davon, dass die Bildung der neuen Knochen bei Resectionen augenscheinlich viel schneller vor sich geht, als unter gewöhnlichen Umständen. Zum Schluss fügt der Autor hinzu, dass er sich des Mittels in Form von Pillen bedient habe.

Indem wir uns mit dieser ausführlichen experimentellen Ar- beit bekannt gemacht haben (von der Zahl der Versuche kann man nicht einmal eine annähernde Vorstellung haben, da in allen Fällen der Autor von "zahlreichen Experimenten" spricht), welche die Wissenschaft augenscheinlich mit höchst interessanten neuen Daten bereichert hat, müssen wir unwillkürlich bedauern, dass sie nicht streng wissenschaftlich dargestellt wurde. Man braucht nur die Knochen junger Thiere in verschiedenen Perioden ihres Wachsthums anzusehen, um sich bald zu überzeugen, welche verschiedenen Schnittbilder wir bekommen, schon abgesehen davon, dass vieles von der mehr oder weniger befriedigenden Entwickelung des Organismus abhängt. Unter solchen Umständen ist der einzige Ausweg der, parallele Beobachtungen an Thieren eines und desselben Wurfes zu machen. Eine solche Untersuchungsmethode wurde von Weguer nur in einer Reihe von Versuchen angewendet und auch da ganz erfolglos. Alle Thiere, die zur Controle dienten, erlagen zufälligen Ursachen, was aber den Autor nicht hinderte, auch hier die erwähnten Schlüsse zu ziehen. Sogar als er bei Hühnern eine höchst interessante Erscheinung fand: eine völlige Ausfüllung des Markkanals unter dem Einflusse der Fütterung mit Phosphor, sagt er nicht eine Sylbe darüber, ob er in 
diesem Falle Controlversuche gemacht hat. Nur erwähnt er ganz nebenbei, dass es ihm gelang, sichtbare Spuren der Wirkung des Phosphors auf das Knochensystem eines Kindes zu bemerken, nachdem das letztere im Laufe von $2 \frac{1}{2}$ Monaten kleine Phosphordosen bekommen hatte, - eine sehr interessante Thatsache, die es verdiente, genauer berücksichtigt zu werden.

Einige Bemerkungen über die Wirkung des Phosphors auf das Knochensystem finden wir in der umfangreichen Arbeit von Korsakow ${ }^{1}$ ), der, indem er 2 Hunden zusammen mit an Kalksalzen armer Nahrung Phosphor in Pillen eingab (der eine Hund bekam in maximo 2, der zweite 4 Pillen täglich, von denen jede 0,00015 reinen Phosphor enthielt, - durchaus keine geringe Dose), Veränderungen in den Knochen hervorrief, die denjenigen gleich waren, welche bei nur an Kalksalzen armer Nahrung beobachtet werden, während Wegner, als er wachsenden Thieren an Kalksalzen arme Nahrung und zugleich minimale Phosphordosen eingab, fand, dass in den Knochen der Thiere an den Stellen, wo bei genügendem Vorhandensein von Kalk in der Nahrung unter der Einwirkung des Phosphors sich ein sehr dichtes Knochengewebe bildet, - beim Fehlen von Phosphor sein histiologisches Aequivalent sich bildet: nehmlich ein sehr dichtes Osteoidgewebe. Leider verfiel der Autor in einen grossen Febler, indem er seinen 2 Hunden je 0,0015 Phosphor eingab ${ }^{2}$ ); es darf daher nicht Wunder nehmen, dass die Thiere bald einer Vergiftung erlagen. Sonderbar ist es aber, dass gerade in diesen Versuchen eine kleine Abweichung von den Angaben Wegner's sich ergab. Dann wurde noch ein Hund mit Phosphor gefüttert mit demselben Endresultat. Dieser letztere Fall ist unter Anderem dadurch interessant, dass der Hund während $2 \frac{1}{2}$ Monaten sehr grosse Dosen von Phosphor ertrug: am Anfang täglich 0,0015 und dann sogar 0,003 mit kleinen Unterbrechungen, und

1) Zur Frage über die Pathogenese der englischen Krankheit. Moskau 1883. Dissert.

2) Die Sache ist nehmlich die, dass bei Wegner ein äusserst fataler Fehler sich einschlich (a. a. O. S. 44), welcher erst nach 12 Jahren durch von Kassowitz verbessert worden ist, - gut, wenn derselbe nur Korsakow in einen Irrthum führte, nicht diejenigen, die das Mittel Kindern eingaben. 
dass er trotzdem nicht erlag; im Gegentheil: der Appetit blieb lange sehr gut. Reninger ${ }^{1}$ ), der die Veränderungen des Knochenmarks bei chronischen Phosphorvergiftungen untersuchte, schenkte bei Sectionen der Veränderung des Knochensystems seine Aufmerksamkeit, dennoch finden wir in den Protocollen seiner Versuche über das Knochensystem nur folgende Angaben: Unter 5 Hunden, welche Phosphor während 1-3 Monaten bekamen, zeigten nur 3 eine Hyperämie des Knochenmarks, während bei den beiden anderen bei der makroskopischen Untersuchung nichts Besonderes gefunden wurde.

Wir sehen also, dass ungeachtet des grossen Interesses der von Wegner angegebenen Daten, seine Arbeit die Aufmerksamkeit der Autoren im Laufe von 12 Jahren wenig beschäftigte. Nur Maas ${ }^{2}$ ) wiederholte im Jahre 1872 seine Experimente über die Wirkung des Phosphors auf den Verknöcherungsprozess und bekam in den Knochen eben solche Veränderungen des Knochengewebes, eben solche Verdichtungen, wie Wegner. Erst im Jahre 1884 erschien die Untersuchung von Kassowitz ${ }^{3}$, wobei dieser, da er den Arbeiten seines Vorgängers volles Vertrauen schenkte, nur einen Theil von Wegner's Versuchen zu wiederholen sich entschloss, bloss in der $\mathrm{Ab}$ sicht, etwas genauer durch mikroskopische Analyse die sog. Phosphorschicht kennen zu lernen, und hauptsächlich um die Frage zu entscheiden, ob diese Schicht ibre Entstehung einer verlangsamten Bildung der Markräume oder einer beschleunigten Bildung von Knochensubstanz bei normal gebildeten Markräumen verdanke. Was die Thatsachen, die durch Wegner dargestellt waren, betrifft, so hielt er es nicht für möglich, an ihrer Richtigkeit za zweifeln.

Die Wirkung der minimalsten Phosphordosen untersuchte Kassowitz an 4 jungen Kaninchen von $6-8$ wöchentlichem Alter, welche den Versuchen während 12, 24, 34, 42 Tagen unterworfen wurden. Bei der makroskopischen Untersuchung der

1) Pathologische Anatomie des Knochenmarks bei Phosphorvergiftung. St. Petersburg 1883. Diss.

2) Tageblatt der Versamming dentscher Naturforseher und Aerzte zu Leipzig. 1872.

3) Die Phosphorbehandlung der Rachitis. Zeitschr. f. klin. Med. 1884. 
Röhrenknochenenden wurde zwischen dem Knorpel und der spongiösen Substanz der Diaphyse ein weisses dichtes Streifchen gefunden, welches nur der Breite nach die normale Lage der hier vorkommenden jungen Knochensubstanz übertraf und lange nicht die Dichtigkeit von compactem Knochengewebe hatte. Bei einer mehr detaillirten Untersuchung dieser sogenannten Phosphorschicht erwies sich, dass sie nichts Anderes darstellte, als eine sehr verbreiterte Zone von entkalktem Knorpel, primären Markräumen und einer metaplastischen Verknöcherung des Knorpels (wir halten uns an die Terminologie des Autors). Was die primären Markkanäle betrifft, so erwiesen sie sich schmäler, als normal; andererseits waren sie sehr weit von einander entfernt, und endlich verzweigten sie sich nicht ihrer ganzen Länge nach, wie es gewöhnlich geschieht. Alle diese Erscheinungen stehen nach Kassowitz' Meinung mit der Verlangsamung des Resorptionsprozesses und der Bildung der Markräume in $\mathrm{Zu}$ sammenhang, und erscheinen als Endresultat der Verlangsamung der Gefässbildung und -Entwickelung. Die Gefässzweige dringen nicht so weit, wie gewöhnlich, in den mit Kalk durchdrungenen Knorpel ein, sind weit von einander gelagert, haben eine engere Lichtung mit einem der letzteren entsprechenden, weniger starken Diffusionsstrom, sind weniger geneigt zur Bildung von Seitenzweigen und endlich, was das wichtigste ist, die Bildung von breiten, mit einander communicirenden Markräumen ist im höchsten Maasse gehemmt. So sucht Kassowitz die Entstehung der Phosphorschicht zu erklären. Aehnliche Bilder bekam er noch an zwei Kaninchen und einigen Hühnern, welche er mitkleinen Dosen fütterte zwecks Vergleichung mit den Resultaten, die beim Füttern mit grösseren Dosen gewonnen wurden. Versuche letzterer Art wurden an 4 Kaninchen und 6 Hühnern gemacht, wobei im Knochenmark folgende Veränderungen entstanden: - Der Zwischenknorpel ist sehr bedeutend erweitert, die sogenannte Phosphorschicht existirt hier zwar schon, aber dem unbewaffneten Auge stellt sie sich als weniger dicht dar, namentlich bei denjenigen Thieren, die verhältnissmässig grössere Dosen bekamen. Bei der mikroskopischen Untersuchung wurde Folgendes gefunden: Die Zone der proliferirenden und hypertrophirten Knorpelzellen war in allen Fällen verbreitert; in einem Falle (wo die grössten 
Dosen gegeben waren) verloren beide Schichten die ihnen eigene Dichtigkeit, weshalb die Säulen der Zellen nach der einen oder anderen Seite hin gelagert erschienen. Zwischen den Säulen der Zellen verliefen Gefässe in der Richtung vom Periost zu den primären Markkanälen, - eine Erscheinung, die bei Rachitis grosse Dimensionen annimmt. Die Zone der Kalkablagerung ist unregelmässig, wellig; die primären Markräume sind in ihren oberen, folglich jüngsten Theilen kolbenartig aufgetrieben, zuweilen buchtig erweitert, in allen Fällen mit Blut überfüllt. Im unteren Theile der Phosphorschicht breite, unregelmässige Markräume. Bei dem Kaninchen, das grosse Dosen erhalten hatte, war diese Schicht durch grosse zusammenfliessende Räume in zwei Theile getheilt, welche nur durch einige schmale Balken verbunden waren, so dass es leicht zur Spaltung kommen konnte. Mit einem Worte, es wurden in dieser Versuchsreihe sehr stark ausgesprochene Bilder gewonnen. Aber eine noch mehr bemerkenswerthe Erscheinung trat bei einem Huhne hervor: noch im Leben begann es schwer zu gehen und konnte sich endlich gar nicht bewegen. Bei der Section wurde eine fast völlige Abtrennung aller Epiphysen der unteren Extremitäten gefunden und überhaupt in den Knochen eine scharf ausgebildete Entzündung. Der Autor brachte unter anderen zum Zweck einer Controlbeobachtung in dieselbe Zelle mit den Thieren, die Phosphor bekamen, ein Huhn, welches keinen Phosphor bekam. Dennoch erwies sich bei der Untersuchung der Knochen, dass sie ein volles Bild der Phosphorschicht ohne Zeichen von Reizung ergaben. Dieser unerwartete Befund wird vom Autor dadurch erklärt, dass das Thier wahrscheinlich minimale Dosen von Phosphor zu sich genommen hatte: entweder beim Picken von Speisen von der Diele, die durch Excremente anderer Thiere verunreinigt war, oder in der Form von Phosphordämpfen aus der von den letzteren ausgeathmeten Luft, und dass das allein schon genïgte, um eine Phosphorwirkung auf die Knochenneubildung hervorzurufen. Man kann nicht behaupten, dass diese Erklärung sehr überzeugend ist für einen vorurtheilslosen Leser: im Ganzen nur ein Controlthier und auch dieses bekam die charakteristische Phosphorschicht. Endlich gelang es bei Hühnern bei fortgesetzter Fütterung mit Phosphor (70 Tage, 3 Monate, 4 Monate) 
mit allmählicher Dosenvergrösserung eine scheinbare Ausfüllung der Markhöhlen zu erhalten; nur füllten sie sich nicht mit compactem Knochengewebe, sondern mit einer unregelmässigen, breitmaschigen, sehr blutreichen Knochenneubildung, welche der Verfasser für das Produkt einer ossificirenden Osteomyelitis hält. Mit einem Worte, grosse Phosphordosen rufen bei Kaninchen und namentlich bei Hühnern leicht einen Entzündungsprozess in den Knochen, im ossificirenden Knorpel und im Knochenmark hervor. Das Phosphorquantum, das keine Reactionserscheinungen hervorrief, war folgendes: für Kaninchen von 6-8wöchentlichem Alter war die tägliche Dosis $15 \mathrm{cmg}$ (Centimilligramm) im Laufe von 12, 24, 34 Tagen, während das Kaninchen, das 42 Tage gefüttert war, wobei es in den letzten 12 Tagen bis zu $30 \mathrm{cmg}$ bekam, schon Reactionserscheinungen an dem Knochengewebe zeigte. Auf diese Weise änderten $300 \mathrm{cmg}$ die Wirkung des Phosphors in ganz entgegengesetzter Richtung.

Scharfe Reactionserscheinungen wurden erhalten, wenn man Kaniuchen (von 500,0 Gewicht) während 20, 30, 40, 60 Tagen täglich je $40 \mathrm{cmg}$ eingab. Eine Abtrennung der Epiphysen erfolgte bei einem Huhne, nachdem es bis zu $20 \mathrm{cmg}$ im Laufe von 44 Tagen erhalten hatte, wobei in den letzten 20 Tagen die Dose bis zu $40 \mathrm{cmg}$ vergrössert wurde. Aus allem eben Mitgetheilten folgt, dass Kassowitz bei weitem nicht in Allem die Befunde Wegner's bestätigte, namentlich nicht in dem Theile seiner Arbeit, wo er die Wirkung der kleinen Phosphordosen untersuchte, die keine Entzündung hervorrufen. Zu allererst erwähnt er nur der Veränderungen der unmittelbar unter dem Zwischenknorpel gelegenen Schicht, während er nichts von Veränderungen in der centralen Knochensubstanz meldet. Was die Veränderungen in der mehrgenannten Schicht betrifft, so bestätigt er auch hier lange nicht Wegner, der die Phosphorschicht als aus wirklich wohl gebildetem Knochen bestehend erklärte, während Kassowitz nichts dergleichen fand und die Phosphorschicht für nichts Anderes ansah, als für eine abnorm verbreiterte Zone mit Kalk durchtränkten Knorpels, primärer Markräume und einer metaplastischen Ossificirung des Knorpels; mit einem Worte aller derjenigen Schichten, die auch normal existiren, nur sind sie vergrössert. Es fragt sich also: wonach soll man 
sich bei der Erkennung der Veränderungen richten und wie sie von der Norm unterscheiden? Das Einzige, wodurch der Sache geholfen werden könnte, wäre, Controlbeobachtungen an gesunden Thieren anzustellen, die aber bei Kassowitz leider ganz fehlen. Ein Versuch wurde freilich in dieser Richtung gemacht, aber auch da wurden bei dem zur Controle bestimmtem Huhne Erscheinungen gefunden, die für Phosphor völlig charakteristisch sind.

Man kann auch nicht umhin, die Aufmerksamkeit darauf zu richten, dass schon ein gar zu kleiuer Unterschied in den Dosen ganz entgegengesetzte Effekte hervorrief.

Wir müssen ferner die Thatsache betonen, dass aus Kassowitz' Arbeit durchaus nicht folgt, dass Phosphor die Eigenschaft besitzt, den Prozess der Knochenneubildung zu verstärken, was gewöhnlich aus seinen Untersuchungen gefolgert wird. In der Einleitung zu seiner Arbeit sagt er ausdrücklich, dass man „die Bildung der Phosphorschicht a priori mit einer Verstärkung und Beschleunigung einer Knochenneubildung innerhalb normal gebildeter und erweiterter Markräume der spongiösen Substanz in Zusammenhang stellen könne"; aber diese Vermuthung findet bei genauer histologischer Untersuchung nicht die kleinste Unterstützung: im Gegentheil, es wird ganz zweifellos, dass die dichte Phosphorschicht in der neu gebildeten Knochensubstanz einzig und allein der beschränkten und verlangsamten Bildung der Markräume ihre Entstehung verdankt. Mit einem Worte, wir haben es nach Kassowitz hier mit einem verlangsamten Prozess der Resorption des jungen Knochengewebes, das unter dem $Z$ wischenknorpel sich bildet, zu thun.

Zum Schluss halte ich es für nöthig zu bemerken, dass ich so ausführlich einige Arbeiten zu besprechen mir nur aus dem Grunde erlaubte, weil die in Journalen und sogar in speciellen Abhandlungen gelieferten Referate über dieselben leider eine zu geringe Vorstellung von dem Inhalt der von mir citirten Arbeiten geben.

Im Februar 1886 machte ich mich selbst an die Erforschung der Wirkung kleiner Phosphordosen auf die parenchymatösen Organe wachsender Thiere. Bei der Section von Hunden, die 3 Monate lang Phosphor bekommen hatten, entschloss ich mich 
aus Wissensdrang, mich genauer mit denjenigen Veränderungen in den Knochen bekannt zu machen, welche den Anstoss zu dem jetzt in der Kinderpraxis bei Rachitis sehr verbreiteten Gebrauch von Phosphor gegeben haben.

Wie gross war meine Verwunderung, als ich bei der Section gar keine Phosphorschicht fand! Das veranlasste mich, meine ganze Aufmerksamkeit auf die Veränderungen der Knochen zu richten.

Zum Versuche wurde gewöhnlich eine schwangere Hündin genommen; als die von ihr gebornen Hündchen 4 bis 5 Wochen alt waren, fing ich an, ihnen Phosphor zu geben. Schon von Anfang der Untersuchungen an überzeugte ich mich, dass jüngere Thiere dazu nicht taugen, da sie zu empfindlich gegen Phosphor sind und oft augenscheinlich minimale Dosen schon Erscheinungen einer chronischen Vergiftung hervorrufen. Gewöhnlich fuhren die jungen Thiere noch 1 bis $1 \frac{1}{2}$ Monate nach dem Beginn des Versuches fort, von Muttermilch sich zu nähren; erst später gingen sie zu gewöhnlicher Nahrung über. Von dem ganzen Wurfe bekam nur die eine Hälfte Phosphor, die zweite blieb zur Controle, wobei ich so wählte, dass in beiden Gruppen einander analoge Individuen der allgemeinen Entwickelung und dem Gewichte nach vorkamen.

Da ich es unbequem fand, so kleinen Thieren Phosphor unter die Haut zu bringen, zog ich es vor, ihnen eine Lösung von Phosphor in Oel in den Mund einzugiessen, umsomehr als ja auch Kindern Phospor in dieser Gestalt verabreicht wird. Pillen verwarf ich schon aus dem Grunde, weil sie schwer von so jungen Thieren heruntergeschluckt werden; andererseits fürchtete ich, dass sie von den Thieren, die Muttermilch bekamen, nicht gut verdaut werden würden. Ueberdies kam der Phosphor in unverändertem Zústande in den Magen. Die Fütterung ging so von statten, dass dem Hunde bei breit geöffuetem Munde auf die Zungenbasis eine Phosphörlösung aufgeträufelt wurde, welche das Thier in meiner Gegenwart herunterschluckte. Wiederholte Beobachtungen überzeugten mich, dass die Thiere dieses Mittel mit grossem Vergnügen zu sich nahmen. (Zur Lösung des Phosphors wurde Mandelöl gebraucht.) Ohne Zweifel kam das ganze Quantum in den Magen. Die Phosphorlösung wurde immer von mir selbst bereitet, ungefähr jeden Monat eine frische. 
Eine abgewogene Menge von weissem krystalliuischem Phosphor wurde, nach sorgfältigem und möglichst sehnellem Abtrocknen mit Filtrirpapier, in ein bestimmtes Quantum Oel gebracht. Die ganz mit Oel gefüllte Dose wurde sorgfältig verschlossen und in einem Wasserbade während einiger Stunden bis zum Siedepunkt des Wassers erhitzt, wobei der Phosphor sich langsam auflöste; auf diese Weise bereiteten wir eine Lösung von $1: 200$ (in Berührung mit der atmosphärischen Luft rauchte sie förmlich und gab einen scharfen Phosphorgeruch), aus welcher wir durch Hinzufügen von Oel eine Lösung von 1: 2000 herstellten. Der letzteren bedienten wir uns bei der Fütterung der Thiere. Bei der Berechnung des Phosphorquantums in einem Tropfen der Lösung wurden die von Reninger gefundenen Daten genommen, die, wie es sich herausstellte, sehr wenig von den Daten von Raymond ${ }^{1}$ ) abweichen, wach welchen ein Tropfen Phosphoröl 0,0208 wiegt. Reninger fand, dass ein Tropfen seiner Lösung $(1: 50) \quad 0,02021$ wog; folglich befand sich in diesem Tropfen 0,00039 reinen Phosphors. Wenn man diese letzte Zahl zu Grunde legt, so findet man, dass ein Tropfen unserer Lösung $(1: 2000)=0,0000097$ reinen Phosphors enthielt (es wird kein grosser Fehler sein, wenn wir der Kürze wegen 0,00001 , d. h. $1 \mathrm{cmg}$ rechnen). Was die Dosirung betrifft, so habe ich mich von folgenden Gesichtspunkten leiten lassen: Die von Wegner vorgeschlagene Dose von $15 \mathrm{cmg}$ $(0,00015)$ ist für junge Hündchen zu gross, und zwar aus folgenden Gründen: einerseits existiren Beweise, dass Hunde gegen dieses Gift empfindlicher sind, namentlich in ejnem so zarten Alter (obgleich das Alter unserer Thiere nur etwas kleiner war, als das bei anderen Autoren); andererseits wollte ich mich den verhältnissmässig kleinen Dosen, die in der Kinderpraxis gegeben werden, nähern. Aus diesem letaten Grunde machte ich folgende Berechnung: die kleinste Dosis, die einem einjährigen Kinde gegeben wird, ist $=50 \mathrm{cmg}(0,00050)$ oder $5 \mathrm{cmg}$ auf jedes kg Gewicht (das Gewicht eines einjährigen Kindes zu $10 \mathrm{~kg}$ angenommen). Die entsprechende Menge für ein Hündchen von 300,0 wäre $1,7 \mathrm{cmg}(0,000017)$; diese Menge von Phosphor ist ungefähr in 2 Tropfen unserer Lösung $(1: 2000)$ enthalten. Mit

1) Journal de Pharmacie. 1884. Févr. 
dem Alter der Hunde wurde die Dose immer vergrössert (in entsprechender Proportion). In der ersten Kategorie unserer Versuche fingen wir nur zum Schluss an, den Thieren, die schon das Gewicht von 2000,0 hatten, täglich bis zu 20 Tropfen unserer Lösung, was $20 \mathrm{cmg}(0,00020)$ des reinen Phosphors entspricht, d. h. etwas mehr, als jene Dosen, die von anderen Autoren täglich Kaninchen von 400-600 g Gewicht gegeben wurden.

Indem wir solche Dosen den Thieren eingaben, sahen wir, dass in ihrem Allgemeinzustande nichts Besonderes zu bemerken war, so lange sie $1 \mathrm{cmg}$ auf $150 \mathrm{~kg}$ Gewicht bekamen; erst als wir zu grösseren Dosen übergingen ( $1 \mathrm{cmg}$ auf 100 Gewicht), bekamen wir eine Verschlimmerung des Allgemeinzustandes. In der zweiten Kategorie der Versuche (zwei Familien von Hunden) fingen wir mit $1: 200,0$ Gewicht an und fütterten die Thiere mit solchen Dosen bis zum Scblusse der Beobachtung. In der dritten Kategorie der Versuche endlich (5 Hunde, 5 Kaninchen und noch ein Hündchen besonders) gaben wir die kleinsten Phosphormengen, nur $1 \mathrm{cmg}$ auf 300,0 Gewicht eines Hundes und eben solche Menge auf 200,0 Gewicht eines Kaninchens. Die Dosis wurde in dieser Versuchsreihe auch bis zum Schlusse der Versuche nicht vergrössert.

Was die Gründe betrifft, die uns veranlassten, die Versuche in drei Kategorien zu theilen, so kam das folgenderweise: Bei der Section der Thiere der ersten Reihe waren wir verwundert, als wir ein volles Bild einer chronischen Phosphorvergiftung fanden, was wir gar nicht bezweckt hatten; deshalb gingen wir zu kleineren Dosen über (die zweite Kategorie), aber als wir uns überzeugten, dass auch hier keine wohlthätige Wirkung des Phosphors im Sinne einer Einwirkung auf das Koochenwachsthum $z u$ bemerken war, sahen wir uns gezwungen, die Dosen noch mehr zu verkleinern (die dritte Kategorie), bei welcher der Phosphor schon als völlig indifferent sich erwies. Während der ganzen Versuchszeit wurden immer Vergleiche zwischen den Thieren, die Phosphor bekamen, und den zur Controle dienenden gemacht, wobei es nicht einmal gelang zu bemerken (in den zwei letaten Kategorien, wo die Thiere bis zum Schluss gesund geblieben sind), dass die Einführung von minimalen Phosphordosen irgend welche günstige Wirkung, soweit das Ergebniss dem 
unparteiischen Beobachter zugänglich ist, erzeuge. Der grösseren Genauigkeit halber haben wir wöchentliche Wägungen der Versuchsthiere angestellt, die wir unten anführen. Was das von einzelnen Thieren aufgenommene Phosphorquantum betrifft, so wurde z. B. ein volles Bild einer chronischen Vergiftung schon durch $600 \mathrm{cmg}(0,00600)$ hervorgerufen, wobei im Laufe von 3 Monaten die tägliche Dosis allmählich von $1 \mathrm{cmg}$ auf 15 stieg; das Hündchen (No. 2 der ersten Kategorie) stieg in dieser Zeit von 326 auf $1355 \mathrm{~g}$ Gewicht. In der zweiten Kategorie, wo Vergiftungserscheinungen schon nicht mehr zu erkennen waren, bekam z. B. der Hund No. 7 im Laufe von 3 Monaten $998 \mathrm{cmg}$. Die tägliche Dosis stieg von 5 auf $15 \mathrm{cmg}$. Das Gewicht des Hundes stieg unterdessen von 1085 auf 3055 .

Endlich in der letzten Kategorie, wo die Thiere bis zuletzt gesund blieben und wo bei der Section kein einziges Organ sich von den Organen normaler Thiere unterschied, nahm z. B. das Hündchen No. $20 \mathrm{im}$ Laufe von $1 \frac{1}{2}$ Monaten $247 \mathrm{cmg}$ ein; die tägliche Dosis stieg von 2 auf $8 \mathrm{cmg}$. Das Gewicht des Hundes erhob sich von 636,0 auf $2750 \mathrm{~g}$.

Aus allem Gesagten kann man schliessen, dass Phosphor in der Menge von $10 \mathrm{cmg}$ auf $1 \mathrm{~kg}$ Gewicht eines Hundes ein volles Vergiftungsbild hervorruft, in der Menge von $5 \mathrm{cmg}$ auf $1 \mathrm{~kg}$ Gewicht die allgemeine Ernährung beeinträchtigt und endlich in der Menge von nur 3,3 $\mathrm{cmg}$ unschädlich bleibt. Die letzten Dosen wurden von uns den Hündchen während 2 Monaten gegeben; welche Folge eine längere Fütterung hervorrufen würde, wissen wir nicht.

\section{Erste Kategorie der Versuche.}

5 vollständig gesunde Hunde, Anfang Februar 1886 geboren, bekommen Muttermilch: 3 erbalten eine Lösung von Phosphor (No. 1, 2 und 3), 2 dienten zur Controle. Der Beginn der Versuche fand am 5. März statt.

Vom 5. März bis zum 21. April einscbl. entwickelten sich alle Hunde gut, waren stets munter, frisch, assen mit grossem Appetit.

Am 21. April wurde bemerkt, dass der Bund No. 1 müde, apathisch wurde, sich immer in dunkle Winkel verkroch; diese Erscheinungen verschwanden jedoch nach 3-4 Tagen ganz und es gelang nicht mebr, etwas Besonderes an ihm zu beobachten. Am 1. Mai wurde wieder bemerkt, dass der Hund No. 1 sich langweilte, sich wie früher in dunkle Winkel verkroch und offenbar weniger als seine Altersgenossen frass; mebr wurde nicht bemerkt. Bis zum 30. April 


\begin{tabular}{|c|c|c|c|c|c|c|c|c|}
\hline atum & $\begin{array}{l}\text { Hund } \\
\text { Ge- } \\
\text { wicht }\end{array}$ & $\begin{array}{l}\text { No. } 1 \\
\text { Zahl der } \\
\text { Tropfen. }\end{array}$ & $\begin{array}{l}\text { Hund } \\
\text { Ge- } \\
\text { wicht }\end{array}$ & $\begin{array}{l}\text { No. } 2 \\
\text { Zahl der } \\
\text { Tropfen }\end{array}$ & $\begin{array}{l}\text { Hund } \\
\text { Ge- } \\
\text { wicht }\end{array}$ & $\begin{array}{l}\text { No. } 3 \\
\text { Zahl der } \\
\text { T'ropfen }\end{array}$ & $\begin{array}{c}\text { Hund No. } 4 \\
\text { Gewicht }\end{array}$ & $\begin{array}{l}\text { Hund No. } 5 \\
\text { Gewicht }\end{array}$ \\
\hline März & 357 & 2 & 326 & 2 & 442 & 3 & 372 & 334 \\
\hline- & 446 & 3 & 378 & 2 & 543 & 3 & 464 & 411 \\
\hline- & 495 & 3 & 406 & 2 & 581 & 3 & 505 & 467 \\
\hline - & 714 & 4 & 570 & 3 & 879 & 5 & 697 & 626 \\
\hline April & 942 & 6 & 717 & 4 & 1145 & 7 & 927 & 808 \\
\hline- & 1141 & 7 & 878 & 5 & 1381 & 9 & 1133 & 985 \\
\hline- & 1322 & 8 & 1140 & 7 & 1717 & 12 & 1337 & 1237 \\
\hline - & 1544 & 11 & 1245 & 10 & 2043 & 15 & 1580 & 1440 \\
\hline - & 1646 & 13 & 1243 & 11 & 2126 & 19 & 1699 & 1509 \\
\hline Mai & 1530 & 15 & 1423 & 11 & 2258 & 21 & 1728 & 1546 \\
\hline- & 1575 & 15 & 1530 & 13 & 2430 & 24 & 1970 & 1640 \\
\hline - & 1635 & 15 & - & 13 & 2780 & 26 & 2175 & 1825 \\
\hline - & 1950 & 16 & 1355 & - & 2985 & 28 & 2280 & 1915 \\
\hline Juni & 1615 & 16 & - & - & 2935 & 30 & 2360 & \\
\hline - & 1700 & 17 & - & - & 3185 & 31 & 2595 & \\
\hline - & 1575 & 15 & - & - & 3320 & 33 & 2775 & \\
\hline- & 1580 & 15 & - & - & 3375 & 33 & 2730 & \\
\hline August & - & - & - & - & 4143 & 41 & & \\
\hline
\end{tabular}

ging die Gewichtszunabme des Hundes ziemlich schnell und beständig vorwärts. Erst am 7. Mai wurde bemerkt, dass der Hund abnabu, aber er unterschied sicb, wie vorber, durch nichts von den anderen. Dann, wie aus der Tabelle zu ersehen ist, fängt sein Gewicht an zu schwanken. Am 20. Juni steht der Hund merklich zurück in seiner Entwickelung. In den letzten 2 Wochen ist der Appetit vermindert, sonst unterschied er sich durch nichts von den anderen. Da der Hund sich in der letzten Zeit offenbar schlecht entwickelte, entschloss ich mich, ihn gleichzeitig mit dem ihm dem Gewicht nach entsprechenden Hunde No. 4, der zur Controle diente, zu tödten. Ueber die Resultate der Section war ich sehr erstaunt: Der Hund No. 1, der Phosphor bekommen hatte, zeigte Folgendes: Das Unterhautfettgewebe schlecht entwickelt, schwach, rosa Schattirung; in der Brusthöhle $100 \mathrm{cem}$ durchsichtiger, seröser Flüssigkeit, von eben solcher Fü̈ssigkeit in der Bauchböhle etwa $200 \mathrm{ccm}$. Die Lungen collabirt, überall etwas Luft enthaltend, meistens dunkelroth gefärbt, keine Verdichtungen, beide Pleurablätter glatt, durchsichtig, ohne Veränderungen. Die Musculatur des Herzens erschlafft, auf der Schnittfiäche deutlich gelbe Nüance. Die Leber von hellbleichgelber Farbe, rosa, dicht, brüchig, knirscht beim Schneiden, ihre Kapsel stark. Die Gallenblase durch eine dicke, dunkelgrüne Flüssigkeit ausgedehnt. Die Theile der Leber, der Därme und des Mesenterium, welche der Blase anliegen, sind gelbgrün gefärbt. In den Nieren ist die Rinde byperämisch, die Marksubstanz bleich, die Nierenoberfläche bleich, die Kapsel lässt sich leicht abziehen. Pankreas bleich, blutarm. Die Schleimbaut des Magens 
und der Därne bleich, von schwachgelber Nüance, mit einer grossen Menge klebrigen Schleims bedeckt. Flüssiges, hellrothes Blut. Das Gewicht der Leber 100,0 .

Die Section des Hundes No. 4 gab ein Bild, das dem eben beschriebenen ganz entgegengesetzt war. Alle Organe normal, das Unterbautfettgewebe reichlich, von weisser Farbe. Eine gelbliche Nüance in den parencbymatösen Organen wurde vermisst. Die Leber weich, von dunkelrother Farbe, ihr Gewicht $167 \mathrm{~g}$.

Der Hund No. 2 bekam Phosphor auch vom 5. Mârz an und entwickelte sich bis zum 14. Mai ganz regelmässig, stets an Gewicht zunehmend, und gar keinen Unterschied vom Controlhund No. 5 darstellend. Vom 14. Mai fingen wir an zu merken, dass der Hund offenbar ohne jeden Grund abzumagern anfing, matt, apathisch wurde und dass sein Appetit merklich vermindert war. Weitere Abweichungen von normalen Functionen gelang es uns nicht, an ihm zu bemerken.

Am 29. Mai wurden die Hunde No. 2 und No. 5 getödtet. Bei der Section des ersteren wurde Folgendes gefunden: Unterhautfettgewebe wenig entwickelt, die Lungen frei, collabirt, entbalten wenig Luft, ungleichmässig gefärbt, in den unteren und hinteren Theilen dunkelroth; die Vorhofshöhlen durch lockere Stücke dunklen Blutes ausgedehnt; die Herzmusculatur zeigt anf der Schnittfäche eine gelbliche Nüance. Die Gallenblase ist mit einer dunkelgrünen, fadenziehenden Flüssigkeit überfüllt. Die Theile der Leber, die der Blase anliegen, grün gefärbt. Die Leber gross, dicht, gleichmässig hellbleichgelb gefärbt. Das Blut überall flüssig, hellrotb. Die Schleimbaut des Magens und der Därme sehr blass, mit gelblicher Nüance. Bei der Section des Hundes No. 5 fanden wir alle Organe normal: Unterbautfettgewebe sebr mächtig, in den parenchymatösen Organen keine Spur von gelblicber Nüance. Die Leber gross, dunkelroth gefärbt, mit Blut überfüllt. In den Gefässen dunkles, dichtes Blut.

Der Hund No. 3 entwickelte sich vom 5. März bis zum 12. August ganz normal, nahm rasch zu und unterschied sich scharf von den anderen Hunden durch seinen sebr guten Ernährungszustand (siebe Tabelle). Am 12. August wurde bemerkt, dass er sich langweilte, sich jmmer in dunkle Winkel verkroch, aber keine weiteren Besonderheiten darbot. Nach 2-3 Tagen erholte er sich ganz. Am 8. September wieder eine Verschlimmerung: er verlor den Appetit, fing an, rasch abzunehmen und wurde apathisch. Getödtet am 10. September. Resultate der Section: Unterhautfettgewebe spärlich, sehr locker; die Lungen stark collabirt, ganz frei, gleicbmässig bleichrosa gefärbt, enthalten wenig Blut, an den Rändern aufgeblasen; einzelne Partien, die einen grossen Theiles eines Lobus einnehmen, ganz luftleer, der Dichtigkeit nach der Haut ähnlich, zusammengeschrumpft, aber keine knotigen Verdichtungen darstellend. Beide Pleurablätter trocken, glatt, obne Trübungen. Das Pericardium fettarm, welk. Die Herzmusculatur bleich, welk, blutarm, zeigt auf der Scbnittfläche eine unbedeutende gelbliche Nüance. Die Leber nicht gross, von dunkler, rothbrauner Farbe; die die Gallenblase 


\section{3}

berührenden Lebertheile hellbraun gefärbt. Das Lebergewebe dicht, schwer zerreissbar (an der Rissstelle ziehen sich von einer zur anderen Seite Fäden). - Die Schnittfläche muscatartig, die Gallenblase durch eine dicke, klebrige, dunkelgrüne Flüssigkeit ausgedehnt. Das Gewebe der Leber knirscht beim Zerschneiden. Die Milz welk. Die Nierenkapsel kann nur mit Mühe abgezogen werden, indem sie Stücke vom Nierenparenchym mit sich nimmt. Die Schleimhaut des Magens und Darms blass, mit schwachgelblicher Nüance.

Die Knochen aller dieser Thiere zeigten bei genauer mikroskopischer Untersuchung absolut keine Abweichung von der Norm.

\section{Zweite Kategorie der Versuche.}

1. 9 Hunde von einer Mutter, alle sehr gut genährt; 5 von ibnen (No.6, 7, 8, 9 und 10) bekamen vom 15. Juli an Phosphor, 2 vom 30. August an, die 2 letzten wurden zur Controlbeobachtung aufbewahrt. Die Resultate der Wägungen und die Zahl der Tropfen der Lösung, die jedem Hunde eingegeben wurden, sind aus folgender Tabelle zu erseben:

\begin{tabular}{|c|c|c|c|c|c|c|c|c|c|c|}
\hline & & & & & & & & & & \\
\hline & & & & & Hund & Hund & & & & \\
\hline & & No. 7 & & & & & & & & \\
\hline & 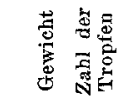 & 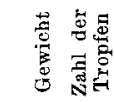 & 蔍 & 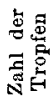 & 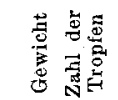 & 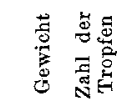 & 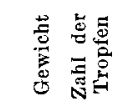 & $\begin{array}{l}\text { ב⿱ } \\
\stackrel{5}{5} \\
0 \\
0\end{array}$ & 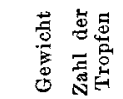 & \\
\hline di & 10625 & 10855 & 991 & 15 & 8864 & $1140 \quad 5$ & $848-$ & 1027 & $1014-$ & 798 \\
\hline & 12816 & 13616 & 166 & 6 & 11255 & 1390 & 039 - & & $266-$ & 1012 \\
\hline & $1510 \quad 7$ & $1484 \quad 7$ & 0 & 7 & 12596 & 1514 & $200-$ & 14 & $01-$ & 1085 \\
\hline ug. & $1850 \quad 9$ & 18509 & 1640 & 8 & 15037 & $1735 \quad 8$ & 1400 & 1995 & $1600-$ & 1210 \\
\hline & 212511 & 231512 & 850 & 9 & 17758 & 226012 & $515-$ & 229 & $915-$ & 1375 \\
\hline pt. & 17958 & 269513 & & 8 & 15357 & 244512 & 7158 & 298 & 19659 & 1865 \\
\hline & $-\quad-$ & 276514 & 1715 & 8 & -- & 256513 & 18359 & 2685 & 02510 & 1885 \\
\hline & -- & 305515 & - & - & - & 276514 & 193510 & .2735 & 223511 & 1800 \\
\hline
\end{tabular}

4 Hunde (No. 7, 9, 12 und 13) boten wäbrend der ganzen Beobachtungszeit gar keine Abweichungen von der Norm dar, indem sie immer zunabmen. Der Appetit blieb bis zum Schluss der Beobachtungen gut. Die Section zeigte absolut keinen Unterschied zwischen den Organen der Thiere, die Phosphor bekommen batten, und denjenigen, die ibn nicht bekamen, ausser dass bei den ersten das Unterhautfettgewebe schlechter entwickelt, welker und weniger weiss war. Die Knochen zeigten nicht die geringsten Veränderungen.

Die übrigen 5 Hunde (No. 6, 8, 10, 11 und 14) entwickelten sich bis zum 17. September ganz normal, nahmen rasch zu und boten gar keine Abweichungen von der Norm dar. Am 18. September erkrankten plötzlich 2 von ibnen (No. 8 und 11) an voller Appetitlosigkeit, starkem Erbrechen und Durchfall und starben nach 2 Tagen. In den nächsten Tagen erkrankten 
noch 3 (No. 6, 10 und 14) und starben auch bald. Bei der Section dieser Thiere wurden ganz gleiche Veränderungen gefunden; deshalb erlaube ich mir nur das Sectionsprotocoll des Hundes No. 8 (vom 21. Sept.) wiederzugeben.

Unterhautfettgewebe wenig ausgebildet. Pleurahöhle frei, die Pleuraoberflächen glatt, durchsichtig. Das Lungenparenchyın blass, blutarm, entbält Blut genug, keine Verdichtungen. Das Pericardium fettarm, die Herzmusculatur blass, welk; die Herzhöblen mit Gerinnseln dicken Blutes überfüllt. Die Leber äusserst welk, blass, leicht zerreisslich, auf der Schnittfläche blutarm; die Grenzen der Läppchen verwischt. Die Gallenblase durch eine grosse Menge dunkelrothgrüner, klebriger, dicker Flüssigkeit ausgedehnt. An der Berührungsstelle der Blase mit der Leber ist die letztere gelblich gefärbt. In den Nieren sind die Rinde und die Marksubstanz scharf von einander getrennt, wobei die erste aus deutlich hervortretenden weisslichen Streifen besteht. Die Kapsel nimmt beim Abziehen Stücke des Nierenparenchyms mit. Die Milz und das Pankreas bieten nichts Besonderes dar. Auf der Magenscbleimbaut subwucöse Hämorrbagien und flache Geschwürchen, beide in beträchtlicher Menge. In den Gedärmen eben solche Hämorrhagien und Geschwüre. Das Blut dunkel, dicht.

Bei der mikroskopischen Untersuchung der Knochen dieser plötzlich gestorbenen Thiere bemerkten wir in ihnen nichts Abnormes; nur entdeckten wir dieselben Veränderungen, wie in den Knochen der übrigen Thiere dieser Kategorie.

2. 3 Hunde (No. 15, 16 und 17), alle von einer Mutter, 5 Wochen alt. Phosphor bekamen nur die 2 ersten. Die Versuche begannen am 4. October

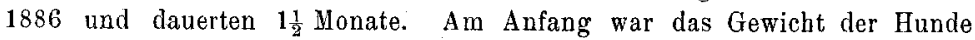
fast gleich (etwa $450 \mathrm{~g}$ ); deshalb fingen wir an, ibnen je $2 \mathrm{cmg}$ täglich zu geben. Die Dosis wurde später gesteigert, aber immer innerhalb bestimmter Grenzen von $1 \mathrm{cmg}$ auf 200,0 Gewicht. Bis zum Schluss der Beobachtungen blieben alle Hunde ganz normal, nur mit dem Unterschiede, dass diejenigen, die keinen Phosphor bekamen, sich etwas besser entwickelten; es gelang nicht, an ihnen krankhafte Veränderungen zu bemerken. Bei der Section erwies sich nur, wie in der vorigen Reihe von Beobachtungen an 9 Hunden, dass die Menge des Unterhautfettgewebes bei den mit Phosphor gefütterten Thieren viel geringer, als bei den zur Controle genommenen, war. Das Fett war etwas weniger weiss. Es. gelang nicht einmal, die kleinste, gelbliche Nüance in der Leber oder in anderen parenchymatösen Organen zu bemerken.

In den Knochen aller Thiere dieser Kategorie wurde nichts Abnormes gefunden.

\section{Dritte Kategorie der Versuche.}

1. Hund No. 18, sehr gut genährt, Gewicht 1320,0 . Der Versuch begann am 25. October 1886 (je $1 \mathrm{cmg}$ auf $300 \mathrm{~g}$ Gewicht). Das Thier nahm 
rasch an Gewicht $\mathbf{z u}$ und am 26. März 1887, als das Thier getödtet wurde, war das Gewicht bis auf $5400 \mathrm{~g}$ gestiegen. Wäbrend der ganzen Beobachtungszeit (5 Monate und 1 Tag) war der Gesundheitszustand des Hundes sehr gut; es wurde kein einziges Mal irgend welche Abweichung ron der Norm bemerkt. Die Phosphormenge, die dem Hunde gegeben wurde, stieg in der letzten Zeit bis zu 18 Tropfen unserer Lösung, was $18 \mathrm{cmg}$ reinen Phosphors entspricht.

2. Eine Hunde-Familie (No. 19, 20, 21, 22, 23), alle gesund und gut entwickelt, wurde 3 Wochen nach der Geburt, am 29. März 1887, dem Versuche unterworfen. Der Phosphor wurde (im Verhältniss von $1 \mathrm{cmg}$ auf $300,0 \mathrm{~g}$ Gewicht) 3 Hunden (No. 19, 20, 21) gegeben, während die 2 anderen zur Controle blieben. Schon bald nach dem Beginne der Beobachtung erwies sich, wie in den vorigen Versucben, dass nicht alle Hunde, die von einer Mutter geboren waren und unter ganz gleichen Bedingungen lebten, sich gleich entwickelten, was jedoch vom Phosphor nicht abhängt, da gewöhnlich diejenigen Thiere in der Entwickelung zurückblieben, die noch vor dem Beginne der Versuche das minimale Gewicht dargeboten hatten. Sie wurden offenbar, kraft irgend welcher Bedingungen, weniger lebensfähig geboren; diesen Umstand muss man bei vergleichender Beobachtung stets im Auge behalten.

In den folgenden Tabellen sind die Resultate der Wägungen und die Phosphordosen angegeben:

$$
\mathrm{T} a \mathrm{~b} \text { e } 11 \text { e } 3 \text {. }
$$

\begin{tabular}{|c|c|c|c|c|c|c|c|c|}
\hline ttum & $\begin{array}{l}\text { Hund } \\
\text { Ge- } \\
\text { wicht }\end{array}$ & $\begin{array}{l}\text { No. } 19 \\
\text { Zahl der } \\
\text { Tropfen }\end{array}$ & $\begin{array}{l}\text { Hund } \\
\text { Ge- } \\
\text { wicht }\end{array}$ & $\begin{array}{l}\text { No. } 20 \\
\text { Zabl der } \\
\text { Tropfen }\end{array}$ & $\begin{array}{c}\text { Hund } \\
\text { Ge- } \\
\text { wicht }\end{array}$ & $\begin{array}{l}\text { No. } 21 \\
\text { Zab] der } \\
\text { Tropfen }\end{array}$ & $\begin{array}{c}\text { Hund No. } 22 \\
\text { Gewicht }\end{array}$ & $\begin{array}{c}\text { Hund No.23 } \\
\text { Gewicht }\end{array}$ \\
\hline März & 496 & 1 & 636 & 2 & 486 & 1 & 626 & 606 \\
\hline April & 566 & 2 & 866 & 3 & 489 & 1 & 896 & 871 \\
\hline- & 661 & 2 & 1171 & 4 & 661 & 2 & 1204 & 1161 \\
\hline - & 911 & 3 & 1506 & 5 & 846 & 3 & 1546 & 1386 \\
\hline - & 1146 & 4 & 1831 & 6 & 1046 & 3 & 2031 & 1706 \\
\hline Mai & 1366 & 4 & 2266 & 7 & 1126 & 4 & 2526 & 2136 \\
\hline- & 1500 & 5 & 2750 & 9 & 1450 & 5 & 3050 & 2600 \\
\hline - & - & - & - & - & 1526 & 5 & & \\
\hline
\end{tabular}

Während der ganzen Beobachtungszeit blieben alle Hunde gesund. Ihr Gewicht stieg allmählich und dem entsprechend wurde auch die Dosis des Phosphors vergrössert. Am 12. Mai wurden 2 Hunde (No. 20 und 23), die dem Gewicht und der allgemeinen Entwickelung nach nahe zu einander standen, secirt. Am 14. Mai wurden noch 2 Bunde (No. 19 und 22) und endlich am 19. Mai der Hund No. 21 secirt.

3. 5 Kanincben (No. 24-28). Der Versuch begann am 7. März 1887, ungefähr 1 Monat nach der Geburt; alle Thiere vollständig gesund. Die von Anderen solchen 'Thieren gegebenen Dosen in Acht nehmend, verordneten auch 
wir ihnen je $1 \mathrm{cmg}$ auf $200,0 \mathrm{~g}$ Gewicht. Solche Dosen wurden bis zum Schluss der Beobachtung gegeben.

T a b e 11 e 4.

\begin{tabular}{|c|c|c|c|c|c|c|c|c|}
\hline & \multicolumn{2}{|c|}{ Kaninchen 24} & \multicolumn{2}{|c|}{ Kaninchen 25} & \multicolumn{2}{|c|}{ Kaninchen 26} & Kaninchen 27 & \multirow{2}{*}{$\begin{array}{l}\text { Kaninchen } \\
\text { Gewich1 }\end{array}$} \\
\hline Datum & $\begin{array}{l}\text { Ge- } \\
\text { wicht }\end{array}$ & $\begin{array}{l}\text { Zahl der } \\
\text { Tropfen }\end{array}$ & $\begin{array}{c}\text { Ge- } \\
\text { wicht }\end{array}$ & $\begin{array}{l}\text { Zahl der } \\
\text { Tropfen }\end{array}$ & $\begin{array}{c}\text { Ge- } \\
\text { wicht }\end{array}$ & $\begin{array}{l}\text { Zahl der } \\
\text { Tropfen }\end{array}$ & Gewicht & \\
\hline 7. März & 198 & 1 & 190 & 1 & 172 & $i$ & 180 & 140 \\
\hline 14. - & 282 & 1 & 285 & 1 & 239 & 1 & 262 & 197 \\
\hline 21. - & 376 & 2 & 375 & 2 & 336 & 1 & 351 & 293 \\
\hline 28. - & 521 & 2 & 520 & 2 & 464 & 2 & 521 & 421 \\
\hline 4. April & 596 & 3 & 631 & 3 & 574 & 3 & 631 & 504 \\
\hline 11. - & 661 & 3 & 706 & 3 & 656 & 3 & 701 & 571 \\
\hline 18. & 691 & 3 & 716 & 3 & 681 & 3 & 736 & 611 \\
\hline $25 . \quad-$ & 741 & 3 & 796 & 4 & - & - & - & 676 \\
\hline 9. Mai & 851 & 4 & 936 & 5 & - & - & - & 836 \\
\hline 16. - & 911 & 4 & 1031 & 5 & - & - & - & 886 \\
\hline
\end{tabular}

Während der ganzen Beobachtungszeit blieben die Kaninchen ganz gesund. Am 22. April wurden 2 davon getödtet (No. 26 und 27), am 19. Mai No. 25 und endlich am 22. Mai die letzten 2 (No. 24 und 28).

Die Ergebnisse der Sectionen bei allen diesen Thieren waren so übereinstimmend, dass wir keine Nothwendigkeit einsehen, sie alle genau anzuführen, um so mehr als alle Organe ganz normal erschienen. In den parenchymatösen Organen wurde keine gelbliche Nüance beobachtet und die Thiere erwiesen sich als ganz gut genährt, indem sie eine bedeutende Schicht von Fettgewebe hatten. Die Knochen der Thiere, die Phosphor bekommen hatten, unterschieden sich durch nichts von den Knochen der Controlthiere.

Wir sehen also, dass in allen 3 Versuchskategorien, wo wir verschiedene Phosphordosen anwendeten, von denen manche ganz unwirksam blieben, andere dagegen das volle Bild einer chronischen Vergiftung durch Phosphor, wie es die Sectionen ergaben, hervorriefen (die Leber der Thiere der ersten Kategorie zeigte bei mikroskopischer Untersuchung eine körnige fettige Degeneration), es uns kein einziges Mal bei der makroskopischen Untersuchung gelang, irgend welche Veränderung in dem Knochensystem zu bemerken. Jenes Streifchen von compacter Knochensubstanz unter dem $Z$ wischenknorpel, von welchem $W$ egner spricht, bekamen wir nicht. Niemals sahen wir auch, dass alles spongiöse Gewebe in der jungen Knochenschicht unter dem Zwischen- 
knorpel durch ein compacteres Gewebe ersetzt worden wäre, wie es bei demselben Autor Bild 7 Tafel II zeigt. Was die Breite der eben erwähnten Schicht betrifft, die nach Kassowitz gerade unter dem Einfluss minimaler Phosphordosen sich verbreitert, so können wir diese Angabe auf Grund unserer Beobachtungen nicht bestätigen, müssen vielmehr darauf aufmerksam machen, dass die Breite dieser Schicht nicht nur bei Thieren eines und und desselben Alters, sondern sogar bei den von einer Mutter gebornen und unter ganz gleichen Verhältnissen lebenden innerhalb der weitesten Grenzen schwankt. Wir erwähnten bereits, dass schon vor dem Beginn der Versuche die Thiere beim Wiegen bedeutende Unterschiede darboten, nach welchen man mit grosser Wahrscheinlichkeit den weiteren Entwickelungsgang eines oder des anderen Hundes voraussagen konnte, vorausgesetzt natürlich, dass keine Nebenumstände, wie Krankheiten, sich einmischten. Nach allem oben Gesagten ist es klar, dass, wenn wir einerseits einen Knochen eines gut entwickelten Thieres, andererseits einen Knochen eines Thieres, das sich aus irgend welchen Ursachen schlecht entwickelte, nehmen, man leicht einen Unterschied in der Länge der Knochen und in der Breite der einen oder der anderen Schicht finden kann. Indem wir die Möglichkeit solcher Fehler in Betracht zogen, nahmen wir immer nur solche Thiere, die ihrer Entwickelung nach sich nahe standen, zum Vergleich (was sich gewöhnlich durch den minimalen Unterschied im Gewicht ausdrückte); unter diesen Verhältnissen fanden wir bei der makroskopischen Untersuchung in den Knochen keinen Unterschied.

Der allgemeine Eindruck ist also der, dass die Knochen aller dieser Thiere sich durch nichts unterscheiden. Als wir uns ferner zum Ziele setzten, die Dicke der Schicht von jungem Knochen, der unter dem $Z$ wischenkorpel liegt, zu ermitteln, sahen wir, dass, wenn es auch Unterschiede giebt, sie jedenfalls sehr klein sind. Mehr oder weniger genau zu messen ist jedoch ganz unmöglich, denn das spongiöse Gewebe schwindet an dem dem Knochenmark zugewendeten Ende nur allmählich, indem es hie und da Inseln zurücklässt. Darum muss man ganz willkürlich einen Punkt wählen und es kann natürlich von Genauigkeit unter solchen Umständen keine Rede sein. Deshalb be- 
schlossen wir, nur die Breite der dichteren, gleich unter dem Knorpel gelegenen Schicht in Zahlen darzustellen. Aber auch hier haben diese Maasse nur einen ungefähren Werth, da der Uebergang der dichteren Schicht in die spongiöse sich ganz allmählich vollzieht. Die Messungen dieser Schicht stellten wir an Längsschnitten des von jedem Thiere genommenen vorderen rechten Oberschenkels an; wir wählten einen constanten Punkt und auf der durch diesen Punkt geführten senkrechten Linie machten wir unsere Messungen. Den Punkt aber bestimmten wir folgendermaassen: Die untere Fläche der Epiphyse dieses Knochens deckt nicht ganz die ihr zugewendete obere Fläche der Diaphyse, so dass zwischen beiden Flächen sich ein Winkel bildet; auf der senkrechten Linie; die durch diesen Winkel geht, maassen wir. Die Zahlen für die Hundefamilie der 3. Kategorie, die von uns ermittelt sind, waren folgende:

$\begin{array}{lcc} & \begin{array}{c}\text { Tab e } 11 \mathrm{e} \\ \text { Breite der } \\ \text { Schicht }\end{array} & \begin{array}{c}\text { Länge des } \\ \text { ganzen Knochens }\end{array} \\ \text { Hund No. 20 } & 4 \mathrm{~mm} & 72 \mathrm{~mm} \\ \text { Hund No. 23 } & 4 \frac{1}{2}- & 72- \\ \text { Hund No. 22 } & 3- & 76 \frac{1}{2}- \\ \text { Hund No. 19 } & 4- & 59- \\ \text { Hund No. 21 } & 1- & 60- \\ \text { Kaninchen No. 27 } & 2 \frac{1}{2}- & 68- \\ \text { Kaninchen No. 26 } & 2- & 68- \\ \text { Kaninchen No. 24 } & 2- & 57- \\ \text { Kaninchen No. } 28 & 1 \frac{1}{2}- & 55-\end{array}$

Aus dieser Tabelle ersehen wir, dass wirklich ziemlich grosse Unterschiede sich herausstellten; aber wenn man zum Vergleich Knochen sich entsprechender Thiere nimmt (z. B. Kaninchen No. 26 und 27 und Hunde No. 20 und 23), so werden die Breiten dieser Schicht freilich auch variiren, jedoch nur um $\frac{1}{2} \mathrm{~mm}$. Indem wir aber das erwägen, was wir früher gesagt, nehmlich, dass man bei der Messung den unteren Punkt willkürlich auswählen muss, so können wir diesen Zahlen durchaus keine entscheidende Bedeatung beimessen; im Gegentheil, sie überzeugen uns, dass diese Schicht gleich breit bei den Versuchsthieren, wie den Controlthieren, ist. 
Zu den mikroskopischen Untersuchungen wählten wir gleichnamige Knochen der einen und der anderen Thiere. Meistentheils dienten uns zur Untersuchung die rechte vordere Hüfte und die linke hintere, wobei wir einen dieser Knochen nur der Länge nach durchsägten und dann zu mikroskopischen Vergleichen in Alkohol brachten; den anderen durchsägten wir auch Anfangs der Länge nach in 2 möglich gleiche Theile, dann trennten wir die Epiphysen sammt einem Theile der Diaphysen bis zum Markkanal ab. Da wir die Absicht hatten, die topographischen Beziehungen der unter dem Zwischenknorpel gelegenen Schichten des jungen Knochens zu erforschen, so mussten wir verhältnissmässig grosse Präparate machen, was die Untersuchung bedeutend erschwerte, da man den Kalk aus ziemlich grossen Knochenstücken ausziehen musste. Zur Erweichung der Knochen nahmen wir folgende Lösungen: Acid. chromicum 0,5-0,3 pCt., Acid. muriat. 0,1 pCt. und endlich Müller'sche Lösung mit Hinzufügung eines kleinen Quantums Ac. mur. (ungefähr bis zu $0,1 \mathrm{pCt}$.). Aber bald mussten wir wegen der nicht völlig günstigen Resultate von den beiden letzteren Flüssigkeiten absehen. In Chromsäurelösung wurden die Knochenstücke entweder bald nach der Section (sorgfältig von Weichtheilen gereinigt) gebracht oder erst, nachdem sie ein wenig zur Fixirung der Elemente in concentrirtem Alkohol gelegen hatten. (Diese letzte Methode erwies sich als besser, aber die Knochen wurden dann in Chromsäurelösung erst nach dem Ansziehen des Alkohols durch Waschen in Wasser während 24 Stunden gebracht). Die Chromsäurelösung wurde fast alle 3-4 Tage gewechselt; sobald wir merkten, dass keine Inseln von compacter Substanz mehr da waren, sistirten wir die Erweichung. Hiebei muss ich bemerken, dass gute Resultate bei dieser Bearbeitungsmethode nur bei der grössten Vorsicht zu erhalten waren, Kaum lag ein Knochenstück in der Lösung einige Tage länger, so wurde es schon $z u$ ferneren Untersuchungeu untauglich, - es kamen dabei äusserst brüchige Schnitte heraus und beim Waschen fielen die in den Maschen der spongiösen Substanz gelegenen Stücke heraus. Die entkalkten Knochenstücke wurden sehr sorgfältig mit fliessendem Wasser während 24-36 Stunden gewaschen, dann wieder auf einige Tage in concentrirten Spiritus 
gebracht, wodurch es erreicht wurde, dass das Präparat in verschiedenen Theilen fast dieselbe Dicke hatte. Mit dem darauf folgenden Einlegen in Gummiarabicum-Lösung waren wir unzufrieden (in Gummi arabicum wurden die Knochenstücke sofort nach der Waschung in Wasser hineingethan), da beim Ausziehen des Gummi arabicum viele Präparate verdorben wurden, weil dabei der ganze Inhalt des spongiösen Gewebes herausfiel. Deshalb gebrauchten wir mehr eine Celloidinlösung (zu gleichen Theilen absoluten Alkohol und Aether), wobei das Präparat, nachdem es 12 Stunden in Spiritus gelegen hatte, in Spiritus, zu dem etwas Aether beigemischt war, übertragen wurde; dann wurde es auf 24 Stunden in eine flüssige Lösung von Celloidin (wodurch erreicht wurde, dass die ganze Dicke des Stückes sich durchtränkte) und für die nächsten 24 Stunden in eine dichtere Lösung gebracht. Aus dem Celloidin herausgenommen und damit auf Korken befestigt, wurden die Präparate in 50procentigen Spiritus gebracht, wo sie für die Untersuchungen conservirt wurden. Zur Färbung benutzten wir Ammoniakcarmin, Hämatoxylin und Pikrocarmin, wobei wir uns überzeugten, dass die Präparate nach langer Bearbeitung mit Chromsäure durch alle diese Farben sehr gut gefärbt wurden, mittelst Carmin und Pikrocarmin aber nur sehr langsam, vorausgesetzt dass alle Chromsäure durch das Auswaschen entfernt war. Am besten war

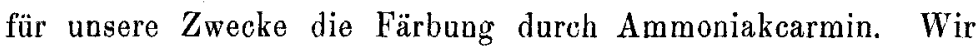
liessen die Schnitte in dieser Lösung etwa 24 Stunden, dann wuschen wir sie in destillirtem Wasser, ohne eine kleine Quantität von Essigsäure hinzugefügt zu haben. Es färbte sich dabei nur das Knochengewebe, so dass schon für das unbewaffnete Auge der Grad seiner Verbreitung gut sichtbar war. Das Pikrocarmin wurde von uns nach dem Recept von Friedländer ${ }^{1}$ ) bereitet. Auch in dieser Farbe hielten wir die Schnitte etwa 24 Stunden; zum Waschen gebrauchten wir dann Wasser, dem einige Tropfen einer gesättigten Pikrinsäurelösung beigemischt waren. Dann brachten wir die Präparate in Glycerin, zu dem auch etwas Pikrinsäure bis zu schwach gelber Schattirung hinzugefügt war. Durch das Hinzufügen von Pikrinsäure zum Wasser und Glycerin erreichten wir eine doppelte Färbung: die Knorpel-

1) Mikroskopische Technik. St, Petersburg 1883, 
zellen; die Elemente des Knochenmarks und das Blut wurden gelb, die Knorpelsubstanz schwach rosa, die Knochensubstanz scharf roth gefärbt. Leider stellte sich diese Färbung als unbeständig heraus: schon nach ein paar Monaten konnte man nicht mehr schöne Bilder, wie gleich nach der Anfertigung der Präparate, bekommen; ein wenig wird diesem Uebelstande durch die Bearbeitung der Präparate mit Salzsäure haltendem Glycerin (nach Friedländer's Rath) abgeholfen. Bei dem endgültigen Hineinthun der Präparate in Canadabalsam fügten wir auch zu dem Spiritus, vermittelst dessen wir das Wasser auszogen, eine kleine Menge von Pikrinsäure zu.

Wir gehen jetzt zu der Beschreibung der bei der mikroskopischen Untersuchung gefundenen Veränderungen über, wobei wir uns an die oben vorgenommene Theilung in 3 Kategorien halten. Die stärksten Veränderungen fanden wir an den Knochen der Thiere der ersten Kategorie. Zu allererst müssen wir bemerken, dass, während beim Betrachten der Knochen mit unbewaffnetem Auge kein Unterschied zu finden war zwischen den Knochen der Thiere, die Phosphor bekommen, und derer, die ihn nicht bekommen hatten, schon an den Schnitten der Unterschied ziemlich scharf hervortritt, was wahrscheinlich davon abhängt, dass hier die Grenzen zwischen den verschiedenen Theilen der Präparate deutlicher zu sehen sind. So fiel es überall in die Augen, dass die Epiphysen bei normalen Thieren länger, die Balken der spongiösen Substanz dicker sind, was hauptsächlich unmittelbar über dem Zwischenknorpel zu bemerken ist. Dann ragt hier der Knorpel in Form eines schwach gefärbten, ganz regelmässigen Streifchens mit ebenen Rändern viel schärfer hervor und seine Dickendimensionen sind viel bedeutender, als diejenigen der Präparate von Thieren, die Phosphor bekommen haben. Bei letzteren unterschied sich der $Z$ wischenknorpel nicht so scharf von dem umgebenden Gewebe und ging unmerklich, namentlich nach unten, in die junge Knochensubstanz über, die ihrerseits beim normalen Thiere durch grössere Dichtigkeit und Mächtigkeit sich auszeichnete. Bei der Untersuchung dieser Präparate unter dem Mikroskope erkannten wir bald, dass die Balken des spongiösen Gewebes bei normalen Thieren eine deutlichere 
Schichtung darbieten (abgesehen davon, dass die Zahl dieser Balken grösser und dass sie viel dicker sind), während bei den Thieren, die Phosphor bekommen hatten, die Substanz der Balken mehr homogen war. Ferner finden wir bei normalen Thiereu öfter der freien Balkenoberfläche anliegende zellige Elemente; die letzteren färben sich sehr gut und sind nicht so dicht angehäuft, wie bei den mit Phosphor gefütterten Thieren, wo die Contouren der einzelnen Zellen sehr unklar werden.

Was für Veränderungen bietet nun der Zwischenknorpel dar? In erster Reihe sehen wir, dass die obere Knorpelschicht (die proliferirende und unveränderte) nicht der ganzen Ausdehnung nach dieselbe Dicke besitzt. An einigen Stellen ist sie fast bis zum völligen Schwinden durch Höhlen, die mit Zellelementen gefüllt sind, ersetzt (sie sieht ganz wie zerfressen aus), wobei unmittelbar über ihr nicht Knochenbalken, wie bei normalen Thieren, sondern schmale Streifen des noch erhaltenen byalinen Knorpels sich finden; die obere Grenze des Zwischenknorpels zeigt deshalb auch sehr unregelmässige Contouren. Dann folgt im normalen Zustande ein Streifen proliferirender Kuorpelzellen, die ganz regelmässige, längs der Knochenaxe sich ziehende Säulenreihen bilden, wobei jede Säule die ganze Höhe dieser Schicht einnimmt. In den Knochen der mit Phosphor gefütterten Thiere gehen die Säulen nicht ganz parallel, oft nehmen sie nur die obere oder die untere Schicht ein; auch ist die Menge des hyalinen Knorpels zwischen den Säulen unzweifelhaft vermehrt, was offenbar mit der verminderten Proliferation der Knorpelzellen in Zusammenhang steht. Endlich erscheint die untere Grenze des Zwischenknorpels bei Thieren, die Phosphor bekamen, nicht so scharf und regelmässig, wie bei normalen. Die Zahl der jungen Knorpelzellen ist bei den Phosphor bekommenden Thieren zweifellos kleiner, als bei normalen; die $Z$ wischensubstanz des Knorpels erscheint nicht so durchsichtig, wie gewöhnlich. Was die Zellen der proliferirenden Schicht anbetrifft, so stehen sie bei den Phosphorthieren in ihrer Grösse zurück, sind vielmehr zusammengeschrumpft, ihre Kerne sind nicht scharf ausgeprägt, endlich färben sie sich schlecht und liegen lange nicht so regelmässig, wie bei normalen Thieren; am meisten geschrumpft sind sie in den untersten Schichten, an der Grenze der Granulations- 
schicht, d. h. in der atrophischen Schicht; mit einem Worte, die Atrophie dieser Zellenreihe ist weit stärker ausgeprägt; während die vorige Schicht, die hypertrophische, weniger von der entsprechenden Schicht normaler Thiere abweicht.

Ich kann nicht umhin, des Umstandes zu gedenken, dass auch bei mir die Angabe Strelzoff's bei der Färbung mittelst Carmin sich bestätigte: nehmlich dass in normalen Knochen die atrophische Schicht sich intensiver färbt, was aber nicht nur von der intensiveren Färbung der Grundsubstanz, sondern auch davon abhängt, dass die Zellelemente dieser Schicht viel besser Farbe aufnehmen. In meinen Präparaten erschienen ausserdem auf der Grenze der hypertrophischen und atrophischen Schicht und auch zwischen dem atrophischen und dem proliferirenden Knorpel zwei schmale, intensiv gefärbte Streifchen, wie künstliche Trennungslinien aussehend, was, wie mir scheint, von einer grösseren Zellenanhäufung an dieser Stelle abhängt.

Alles eben Beschriebene wurde von uns an den Knochen normaler Thiere beobachtet; in den Knochen der Phosphor bekommenden Thiere dagegen waren nur Spuren von diesen Bildern zu sehen. -

Wir kommen jetzt zu der Granulationsschicht. Hier finden wir bei den Phosphorthieren folgende Abweichungen von der Norm: Wie schon erwähnt, erscheint die Grenze dieser Schicht gegen die höher gelegenen sehr unscharf und unregelmässig; diese Schicht färbt sich schlecht in Carmin, was besonders an der Grundsubstanz des hyalinen Knorpels zu bemerken ist. Bei schwacher Vergrösserung sieht man hier nicht jene buchtige Erweiterung der primären Markräume in ihren oberen Theilen, wie sie bei der Betrachtung von Knochen normaler Thiere in die Augen fällt. Die Zahl der Zellenelemente dieser Schicht ist bedeutend vermindert, namentlich in den obersten Theilen, wo noch keine grossen sinuösen Räume sich auszubilden Zeit hatten. Was die Zellen selbst anbetrifft, so sind es meist kleine.

Endlich erwies sich die Höhe dieser Schicht, namentlich ihr oberer Theil, wo noch keine sinuösen Räume vorhanden sind, kleiner; dabei merkten wir hier nicht selten, dass die obengenannten sinuösen Räume bis zur atrophischen Schicht des Knorpels reichten, was im normalen Zustande niemals zu beobachten ist. 
Indem wir alle von uns in den Knochen der Thiere der ersten Kategorie, die Phosphor bekommen hatten, gefundenen Veränderungen resumiren, sehen wir, dass in der Zone der endochondralen Entwickelung des Knochens einzelne Schichten bedeutend vermindert und nicht so regelmässig, wie im normalen Zustande, gelagert sind, ferner dass die Zwischensubstanz des Knorpels sich relativ schlechter färbt und dass endlich die Zellenelemente kleiner und die Kerne in ihnen weniger ausgeprägt sind. Was die Zellen der atrophischen Schicht betrifft, so erscheinen sie mehr geschrumpft, - wir haben es mit einem Worte mit einer einfachen Gewebsatrophie zu thun.

An den Knochen der Thiere der zweiten Kategorie, d. h. derjenigen, welche weniger Phosphor bekommen hatten, erhielten wir lange nicht so scharfe Bilder, wie die eben beschriebenen, namentlich im Bereiche des Zwischenknorpels, wo die einzelnen Schichten markanter sind, wenn auch nicht so, wie bei normalen Thieren. Niemals wird hier eine schiefe Richtung der Knorpelzellensäulen in der proliferirenden, hypertrophischen und atrophischen Schicht, wie das oben von uns beschrieben worden ist, gefunden. Die Zellenelemente aller Schichten unterscheiden sich durch nichts Besonderes; nur die Höhe der Schichten war bei diesen Thieren merklich vermindert, was hauptsächlich im Bereiche der hypertrophischen und atrophischen Schicht in die Augen fiel.

Offenbar kann bei diesen Thieren von atrophischen Erscheinungen nicht mehr die Rede sein; man kann nur sagen, dass die Entwickelung ihrer Knochen nicht so gut, nicht mit derselben Energie vor sich geht, wie bei normalen Thieren.

Endlich in der dritten Kategorie unserer Versuche konnten wir bei der genauesten Untersuchung keinen Unterschied zwischen den Knochen derThiere, die Phosphor bekommen hatten, und denen normaler Thiere auffinden, - sie waren in allen Beziehungen vollkommen gleich. Die grösste Aufmerksamkeit lenkten wir dabei auf die unter dem $Z_{\text {wischenknorpel befindliche Schicht des }}$ jungen Knochens, aber auch hier fanden wir jene Veränderung, die Kassowitz so genau beschreibt, nicht. Nur muss man es so machen, wie wir es oben beschrieben haben, nehmlich zu vergleichenden Beobachtungen nur Knochen solcher Thiere nehmen, die der allgemeinen Entwickelung nach gleich sind. Wir sprechen 
nicht weiter davon, was für ein colossaler Unterschied zuweilen zwischen Thieren eines und desselben Wurfes herauskam, die unter gleichen Verhältnissen lebten und während der ganzen Beobachtungszeit an keiner Krankheit litten. Aus der letzten Tabelle ist zu ersehen, dass die Maasse eines und desselben Knochens bei Hunden der letzten Kategorie zwischen 59 und $76 \frac{1}{2} \mathrm{~mm}$ sch wankten. Es ist klar, dass wenn man diese beiden Knochen nehmen und die Dimensionen der einen oder anderen Schicht vergleichen würde, ein ganz bedeutender Unterschied heranskommen müsste, aber es ist leicht einzusehen, dass das ein Irrthum wäre. Hierbei wollen wir jedoch erwähnen, dass bei einem von 11 Thieren der letzten Kategorie (Hund No. 20) wir wirklich bemerkt haben, dass es sich vor den anderen Thieren durch bessere Entwickelung seines Knochensystems auszeichnete, was sich darin ausdrückte, dass die Granulationsschicht dichter erschien und die unregelmässigen sinuösen Räume etwas niedriger, als gewöhnlich, lagen. Aber wenn wir uns erinnern, dass dieser Hund vor allen anderen durch eine äusserst gute Entwickelung, die auch später stets in die Augen fiel, sich auszeichnete, so wird es klar, weshalb in diesem Falle die Entwickelung des Knochensystems besser, als in den anderen Fällen, vor sich ging. Diesen Unterschied der Wirkung des Phosphors zuzuschreiben, hätten wir absolut kein Recht, da wir bei den anderen Thieren dieser Kategorie, die Phosphor bekommen hatten, nichts dergleichen fanden.

\section{Schlüsse.}

1. Der Phosphor ist ein viel stärkeres Gift, als für welches man ihn gewöhnlich hält.

2. Beim Dosiren dieser Substanz für einen jungen Organismus ist es nicht genügend, die Dosis entsprechend dem Gewichtsunterschiede zu verkleinern.

3. Es giebt keine Phosphordosen, die einen günstigen Einfluss auf den wachsenden Knochen ausüben.

4. Eine unbedeutende Verdaungsstörung, die beim Gebrauch minimaler Phosphordosen entsteht, kann eine fatale Bedeutung haben.

5. $10 \mathrm{cmg}$ Phosphor auf $1 \mathrm{~kg}$ Gewicht rufen bei jungen Hunden das volle Bild einer chronischen Vergiftung mit scharf 
ausgeprägten Erscheinungen eines atrophischen Prozesses an den Ablagerungsstellen des jungen Knochens hervor. $5 \mathrm{cmg}$ auf $1 \mathrm{~kg}$ Gewicht beeinträchtigen die normale Entwickelung des jungen Organismus. Erst $3,3 \mathrm{cmg}$ auf $1 \mathrm{~kg}$ bleiben ganz unschädlich.

6. Kein einziges Mal gelang es mir, ein Bild zu erbalten, das den von Wegner seiner Arbeit (a. a. O. Taf. II. Fig. 5, 6 and 7 ) beigefügten Bildern ähnlich gewesen wäre.

7. Phosphordosen, die als ganz unschädlich sich erwiesen, übten offenbar keinen Einfluss auf den allgemeinen Gesundheitszustand der Thiere aus.

8. Bei der chronischen Vergiftung durch ganz kleine Phosphordosen bekamen wir (was schon von anderen Autoren betont worden ist) eine ausgesprochene Volumensverminderung der Leber mit offenbar gesteigerter Bindegewebswucherung (sie knirschte beim Schneiden, war schlecht zerreisbar).

9. Es giebt keinen Grund zum Phosphorgebrauch bei Knochenkrankheiten.

\section{Zusatz des Herausgebers.}

Die Abhandlung des Herrn $\mathrm{K}$ issel hat so sehr die Zeichen einer zuverlässigen und auf wohl überlegten experimentellen Untersuchungen beruhenden Darstellung, sie weckt zugleich von Neuem die Aufmerksamkeit für so wichtige Verhältnisse, dass ich trotz nicht geringer Bedenken ihre Veröffentlichung übernommen habe. In diesem Entschlusse bin ich auch dadurch nicht wankend geworden, dass das Gesammtergebniss in diametralem Gegensatze zu den, unter meinen Augen gewonnenen Resultaten des Herrn Georg Wegner steht. Aber eben so wenig hat Herr Kissel meine Meinung über diese letzteren erschüttert. Herr Wegner hat seine Experimente zu einer Zeit ausgeführt, wo er mein Assistent war. Die Beweisstücke für seine Angaben, die in diesem Archiv 1872. Bd. 55. S. 11 u. fg. und 1874. Bd.61. S. 69 und 73. Taf. $V$ veröffentlicht sind, befinden sich noch heute in der Sammlung des Pathologischen Instituts, und es gewährt mir eine besondere Freude, sie meinen Zuhörern und den Besuchern des Instituts immer von Neuem zeigen zu können. Sie gehören in der That zu den Glanzstücken unseres Museums. 
An ihrer Beweiskraft zu zweifeln, scheint mir trotz der Arbeit des Herrn Kissel unmöglich. Da ich aber auch nicht berechtigt bin, die Zuverlässigkeit dieses Beobachters in $Z$ weifel zu ziehen, so bleibt mir nichts übrig, als meine Herren Mitarbeiter aufzufordern, den Grund der Differenz durch weitere Experimente zu ermitteln. Natürlich verzichte ich in einer Frage von so grosser principieller Wichtigkeit nicht darauf, im Pathologischen Institut neue Untersuchungen zu veranlassen, aber ich bin gern bereit, anderen Experimentatoren zunächst keine Concurrenz zu machen. Rudolf Virchow.

\section{VI. \\ Blutveränderungen bei Anämien.}

(Aus dem chemischen Laboratorium der medicinischen Klinik des Herrn Prof. Dr. H. Eichhorst in Zürich.)

Von Sophie v. Moraczewska aus Warschau.

(Hierzu Taf. IV und V.)

Das Blut wurde in seinen pathologischen Zuständen am frühesten in Bezug auf das Verhältniss zwischen Hämoglobingehalt und Blutkörperchenzahl untersucht. An der Lösung dieser Frage ist am meisten gearbeitet worden, - sie ist diejenige, welche zu den klinischen Untersuchungsmethoden gehört -, und ihre Resultate sind am besten bekannt.

Das Verhältniss zwischen Hämoglobingehalt und Blutkörperchenzahl ist, nach Untersuchungen von $W_{\text {elker }}{ }^{1}$ ), Worm. Müller ${ }^{2}$ ) und Malassez ${ }^{3}$, bei Gesunden ein constantes. Ein Sinken des H-Gehaltes der Blutkörperchen wurde in pathologischen Zuständen zuerst von $W$ elker ${ }^{4}$ ) (bei Anämien), dann

1). Vierteljahrschr. f. prakt Heilk. Prag 1854. H. 4. 11. - Zeitschr. f. prakt. Med. 1863. Ser. III. 20. 257.

2) Citirt nach Maly's Ber. VII. 102.

3) Archives de physiol. 2. Ser. 4. I. - Gaz. méd. de Paris. p. 516.

4) a. a. 0 . 